\title{
Edge Detection of Metal Thickness of Electromagnetic Acoustic Transducer Based on Super-Heterodyne Phase- Sensitive Detector
}

\author{
Zhichao CAI ${ }^{\mathrm{a}, 1}$, Zhenyong $\mathrm{ZHAO}^{\mathrm{a}}$,Lan $\mathrm{CHEN}^{\mathrm{a}}$, and Guiyun TIAN ${ }^{\mathrm{b}}$ \\ ${ }^{a}$ School of Electrical and Automation Engineering, East China Jiaotong University \\ ${ }^{b}$ School of Engineering, Newcastle University, Newcastle, UK, NE1 7RU
}

\begin{abstract}
In order to improve the thickness measurement accuracy of metal plate, a new method based on phase detection thickness is proposed. Two modes of ultrasonic echo are generated by electromagnetic acoustic transducer (EMAT) at the edge of specimen, and super-heterodyne phase-sensitive detection technique is used to collect the phases of the ultrasonic, then the thickness information of the tested specimen is obtained accurately. Firstly, the finite element model of EMAT is built, and the time-domain characteristics of acoustic echo are analyzed. Secondly, the $0^{\circ}$ and $90^{\circ}$ phase detection on echo signal are detected by the super-heterodyne phasesensitive detection system. Finally, the relationship between phase information and specimen thickness is analyzed. The results show that the detection method, which based on super-heterodyne phase-sensitive detector of edge mode conversion, can realize the detection of metal plate thickness, and the phase error range is controlled within $0.23 \%$.
\end{abstract}

Keywords. edge detection, super-heterodyne phase-sensitive detector, EMAT, finite element analysis

\section{Introduction}

In general, ultrasonic testing method is used for the thickness measurement of metal materials, the principle of piezoelectric ultrasonic energy exchange is often used to produce ultrasonic wave for the detection of solid materials. But piezoelectric transducers (PZT) needs to be coated with coupling agent in the detection, and the testing specimen needs to be pretreated, so this kind of direct contact detection method requires higher detection environment, higher detection cost and lower detection efficiency [1]. Besides, PZT usually excites primary wave (P wave), but the shear wave ( $\mathrm{S}$ wave) with the same frequency has better resolution detection, so the higher resolution detection can only be achieved by applying higher excitation frequency.

Compared with PZT, the electromagnetic acoustic transducer (EMAT) has the characteristics of non-contact detection, no coupling agent and no pretreatment on the specimen surface in testing [2], at the same time, EMAT is more likely to excite S wave, under the same resolution detection, the excitation frequency of EMAT is only half of

\footnotetext{
${ }^{1}$ Corresponding Author, Zhichao Cai, E-mail: czchebut@foxmail.com
} 
that of PZT [3]. Therefore, the research of electromagnetic ultrasonic testing technology based on EMAT has been more extensive in recent years [4].

There are two main methods for measuring metal thickness by EMAT: 1. Pulse-echo method; 2. Electromagnetic acoustic resonance (EMAR) method. In the pulse-echo method, the thickness of metal specimen is calculated by using the peak data of two echo signals[5], when the metal thickness is relatively thick, the echo signal will be very weak, higher requirements are put forward for using pulse echo method to accurately measure echo signal. In the EMAR method, which has a high detection efficiency, the damage evaluation of $2.5 \mathrm{~mm}$-thick aviation aluminum plate can be carried out accurately by using EMAR method [6]. However, it is still difficult to the peak data due to the influence of acquisition frequency and noise [7]. Moreover, the EMAR method needs to increase the number of excitation cycles to realize the superposition of adjacent echoes, this will cause strong main pulse interference, so this method is often used in measuring sheet metal [8].

Based on the above shortcomings, a detection method based on ultrasonic edge detection and super-heterodyne phase-sensitive detector is proposed in this paper, accurate phase information is used to feedback thickness information of specimen. The rest of the paper is organized as follows : Section 2 introduces the principle of EMAT detection at the edge of the specimen. The principle of super-heterodyne phase-sensitive detector is demonstrated in Section 3. Section 4 introduces finite element modeling simulation and analysis, followed by the discussion in Section 5.

\section{The principle of EMAT detection at the edge of the specimen}

The edge detection of EMAT is similar to the principle of traditional EMAT thickness measurement, the difference is that EMAT is placed on the edge of metal material to produce multi-mode ultrasonic. When a high frequency excitation pulse current is fed into the coil, the eddy current will be induced in the skin depth of the metal surface, and the vibration of those particles are affected by Lorentz force under the static magnetic field provided by $\mathrm{Nd}-\mathrm{Fe}-\mathrm{B}$ permanent magnet, $\mathrm{S}$ wave and $\mathrm{P}$ wave propagating downward are generated in the interior of the specimen, Rayleigh wave ( $R$ wave) propagating along the surface is generated on the side of the specimen.
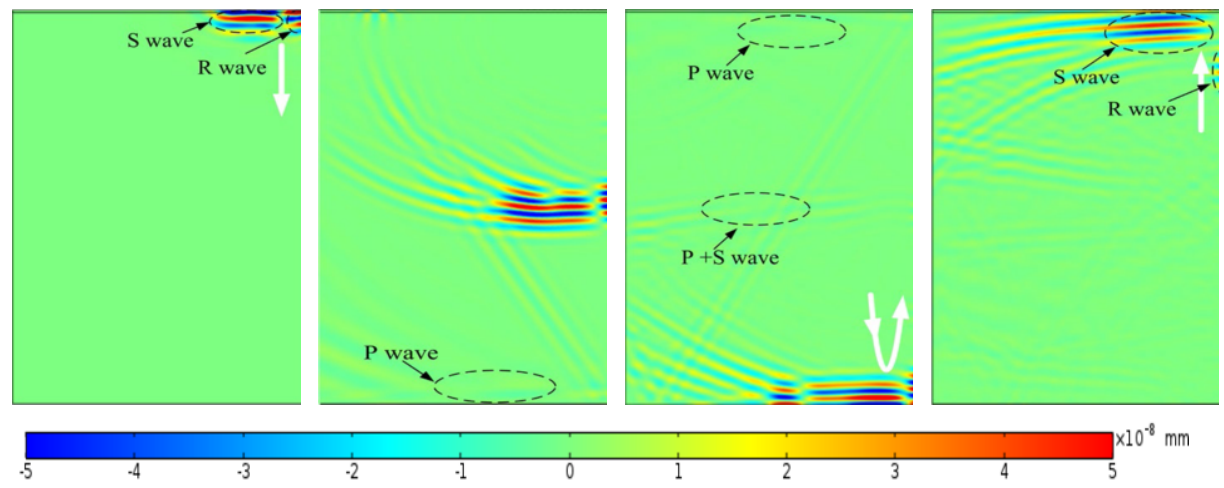
(a) $\mathrm{t}=1 \mu \mathrm{s}$
(b) $\mathrm{t}=6 \mu \mathrm{s}$
(c) $\mathrm{t}=11 \mu \mathrm{s}$
(d) $\mathrm{t}=20 \mu \mathrm{s}$

Figure 1. The propagation process of ultrasonic wave in the specimen 
To simulate the ultrasonic propagation process, a two-dimensional finite element model of electromagnetic ultrasonic transceiver is established, the frequency of electromagnetic ultrasonic excitation $F_{S}=3 \mathrm{MHz}$, The displacement distribution of ultrasonic wave at different time in $\mathrm{Y}$-axis direction is shown in Figure 1. At the beginning, EMAT excites both $\mathrm{S}$ wave and $\mathrm{R}$ wave on the surface of metal specimen, because ultrasonic wave speed of different modes is different, with the increase of propagation time, two modes of ultrasonic are gradually separated. When the ultrasonic wave hits the bottom of the specimen, it will reflect the ultrasonic wave and propagate to the top of the specimen according to the original path, and the magnetic flux in the coil will be changed. According to Faraday's electromagnetic induction law, the time domain voltage signal containing two modes of ultrasonic echo will be induced in the coil.

According to the induced voltage signal received by the coil, and through the mathematical relationship:

$$
\Delta T=\frac{2 n \cdot\left(V_{S}-V_{R}\right) \cdot D}{V_{S} \cdot V_{R}}, n \in N^{+}
$$

the time $\Delta T$ between the peak value signal of adjacent $\mathrm{S}$ wave and $\mathrm{R}$ wave can be calculated. Where, $V_{S}$ is $\mathrm{S}$ wave velocity, $V_{R}$ is $\mathrm{R}$ wave velocity, $n$ is the positive integer, $D$ is the thickness of the specimen.

\section{The principle of super-heterodyne phase-sensitive detector}

In general, the thickness information of the specimen can be fed back through formula (1), but the high noise and low detection efficiency of electromagnetic ultrasonic hinder the accurate extraction of the time-domain signal. However, the super-heterodyne phasesensitive detection method can effectively extract the echo signal by improving the signal-to-noise ratio, and obtain more accurate detection signal information. As shown in Figure 2.

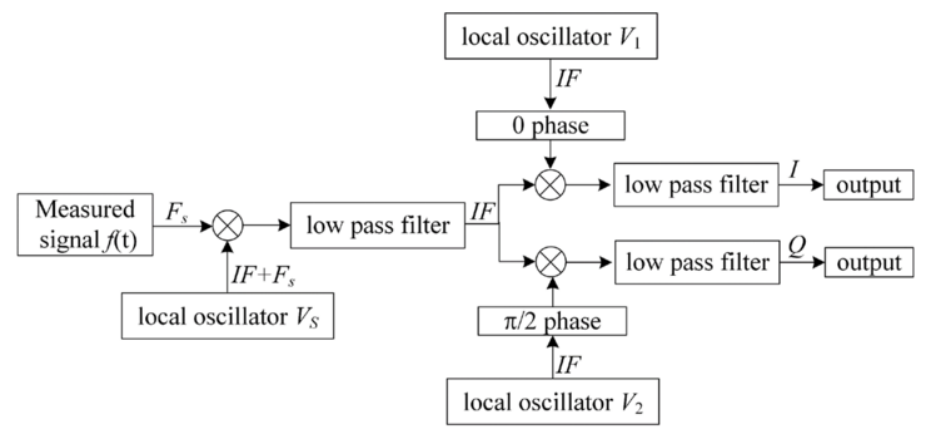

Figure 2. The principle of super-heterodyne phase-sensitive detector

The measured signal is regarded as a sinusoidal pulse signal $f(t)$ with Gaussian window modulation with exciting frequency $F_{S}$ :

$$
f(t)=A_{\mathrm{t}} e^{-\alpha(t-\tau)^{2}} \sin \left[2 \pi F_{s} t+\varphi\right]
$$


where $A_{\mathrm{t}}$ is the excitation current amplitude, $\alpha$ is the bandwidth coefficient, $\tau$ is the time coefficient, $\varphi$ is the phase angle of the measured signal.

The local oscillator generates a local oscillation signal $V_{S}$ with frequency of $I F+F_{S}$, $f(t)$ is mixed with $V_{S}$ through the mixer, and the mixed signal contains two frequencies of $I F$ and $I F+2 F_{S}$, when the mixed signal passed through the low-pass filter, intermediate frequency signal with frequency $I F$ is only retained. According to the principle of superheterodyne phase-sensitive detector, two reference oscillation signals $V_{l}$ and $V_{2}$ with frequency $I F$ and phase difference $\pi / 2$ are generated by the local oscillator. They are multiplied with intermediate frequency signal by multiplier in order to obtain the same phase signal and orthogonal signal. These two signals are respectively filtered through the low-pass filter to remove the high frequency signals, and the differential frequency signals $I$ and $Q$ are retained and output.

$$
\begin{aligned}
& I=A_{g} e^{-\alpha(t-\tau)^{2}} \sin \varphi \\
& Q=A_{\mathrm{g}} e^{-\alpha(t-\tau)^{2}} \cos \varphi
\end{aligned}
$$

$A_{g}$ is constant, including the total gain and conversion coefficient. According to formula (3) and formula (4), the phase $\varphi$ of the received signal is deduced:

$$
\varphi=\arctan \frac{I}{Q}
$$

\section{Finite element simulation and analysis}

Finite element simulation software is used to simulate the ultrasonic field of EMAT, the echo voltage signal is received in the spiral coil as shown in Figure 3(a).

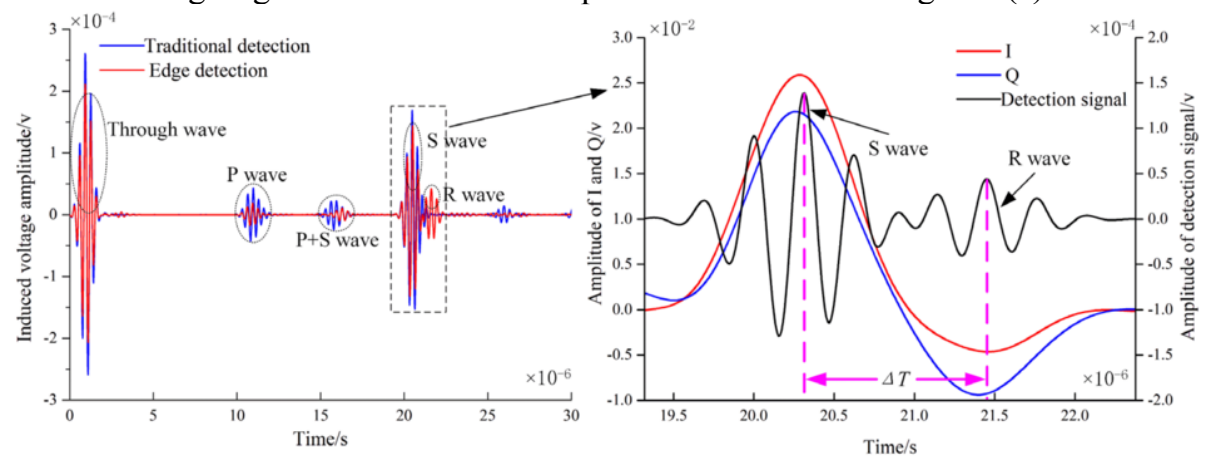

Figure 3. (a).Received time domain signal (b). Super-heterodyne phase-sensitive detection

According to the formulas (2) to (4), the $\mathrm{S}$ wave and $\mathrm{R}$ wave signals are processed through the super-heterodyne phase-sensitive detection system, and the result is shown in Figure 3(b).

When the thickness of the specimen change slightly, it is difficult to determine thickness change information by pulse-echo method. However, the phase change is very 
significant after the phase detection of $0^{\circ}$ and $90^{\circ}$, because for every $0.1 \mu$ s increase in the time, the phase angle increase by 108 degrees after the super-heterodyne phasesensitive detection. Therefore, the change in phase information can be used to reflect the change in specimen thickness accurately. But the phase angle $\varphi_{S}$ of $S$ wave and phase angle $\varphi_{R}$ of $\mathrm{R}$ wave are both between $\left(-90^{\circ}, 90^{\circ}\right)$, in order to determine their true phase relationship, the $\mathrm{S}$ wave and $\mathrm{R}$ wave data are processed, as shown in Figure 4. Where, $j$ is $\mathrm{S}$ or $\mathrm{R}$.

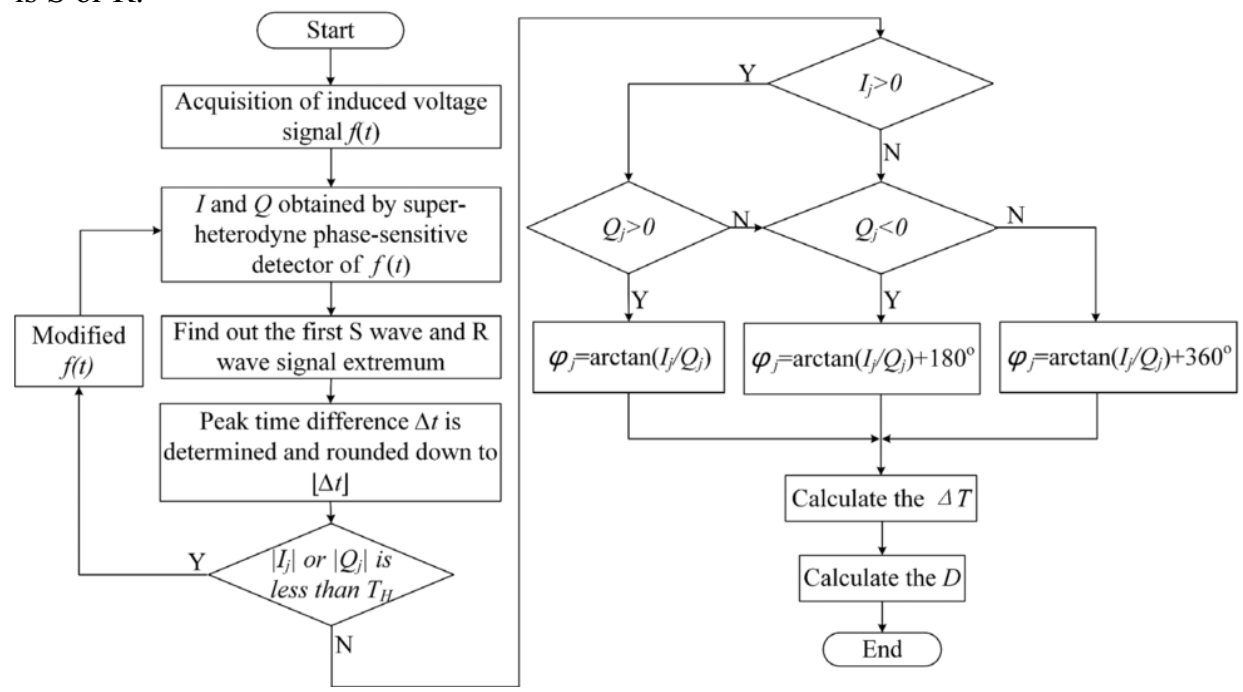

Figure 4. Data processing flow chart

There is the echo noise interference in the output signals $I$ and $Q$ by the superheterodyne phase-sensitive detection system, which makes the phase extremum of the noise generated in addition to the $\mathrm{S}$ wave and $\mathrm{R}$ wave. Finally, this will lead to inaccurate extraction of the extremum of the signals $I$ and $Q$. In order to solve this problem, the threshold $T_{H}$ is set in this paper, $T_{H}$ is generally not less than 0.6 times the maximum value of $I$ or $Q$ between $\left(-90^{\circ}, 90^{\circ}\right)$.It means that when the extreme value of $\left|I_{j}\right|$ or $\left|Q_{j}\right|$ is lower than $T_{H}$, the induced voltage signal $f(t)$ needs to be corrected. The specific correction method is to change the initial phase of the excitation signal, because the signal $f(t)$ is affected by the initial phase of the excitation signal. Finally, the extremum of the output signal $I$ and $Q$ will be changed to meet the threshold $T_{H}$.

According to the phase signal $I$ or $Q$ of the adjacent $\mathrm{S}$ wave and $\mathrm{R}$ wave, the peak time $\Delta t$ is determined and rounded down to $\lfloor\Delta t]$. From the phase angle relation:

$$
\Delta T=\frac{\varphi_{R}-\varphi_{S}+2\lfloor\Delta \mathrm{t}\rfloor \pi}{2 \pi F_{\mathrm{s}}}
$$

the time $\Delta T$ can be obtained, and the thickness $D$ can be calculated accurately.

In order to verify the feasibility of the super-heterodyne phase-sensitive detection method, aluminum material in this paper is taken as the research object, its geometric structure is shown in Figure 5(a), which is used to simulate the tiny change of thickness of material due to uneven friction. Then the phase value $\varphi$ calculated by sine signal $I$ and cosine signal $Q$ is compared with the theoretical phase value, and its phase error curve is 
shown in Figure 5(b). The results show that the maximum relative error of phase value is $0.23 \%$, and the maximum error of aluminum thickness is $0.077 \mathrm{~mm}$.
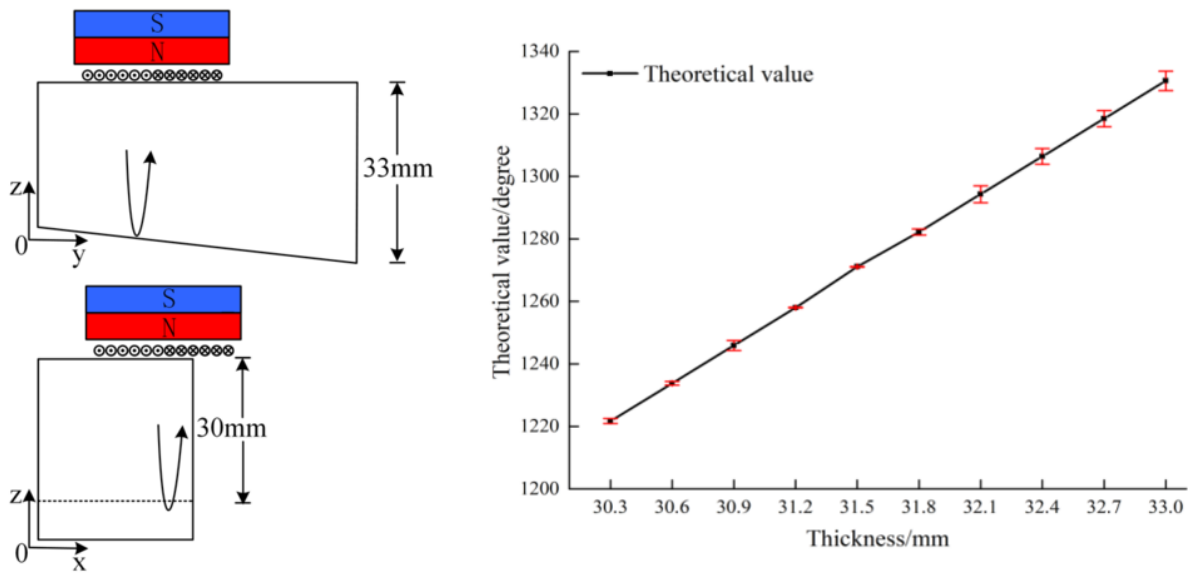

Figure 5. (a) Geometry of specimen, (b) Phase error curve

\section{Conclusions}

In this paper, a method based on super-heterodyne phase-sensitive detector of edge mode conversion is proposed to measure specimen thickness. The results show that the method has a higher accuracy for the thickness, and a higher resolution for the specimen with slight changes of thickness. This method is superior to the traditional pulse-echo method, and the results prove the potential of this method in thickness measurement. However, the $\mathrm{R}$ wave signal is weak in the simulation process. Further research will be conducted on how to optimize the transducer to enhance the detection effect.

\section{References}

[1] Putkis O., Dalton R.P., Croxford A.J., The anisotropic propagation of ultrasonic guided waves in composite materials and implications for practical applications, Ultrasonics 65 (2016),390-399.

[2] Peng Chen, Delai Han, Qiangfu Cai, Meiquan Liu, Research progress in electromagnetic acoustic testing, Foreign Electronic Measurement Technology 31 (2012),18-21.

[3] Shujuan Wang, Riliang Su, Xiaoyang Chen, Lei Kang, Guofu Zhai, Numerical and experimental analysis of unidirectional meander-line coil electromagnetic acoustic transducers, IEEE Transactions on Ultrasonics, Ferroelectrics, and Frequency Control 60 (2013),2657-2664.

[4] Peng Chen, Delai Han, Qianfu Cai, Meiquan Liu, Research progress in electromagnetic acoustic testing, Foreign Electronic Measurement Technology 31 (2012), 18-21.

[5] Jingpin Jiao, Zhaoqing Yu, Wenhua Liu, Qiang Zhang, Electromagnetic acoustic thickness measurement method of thin liquid layer based on wavelet transform , Chinese Journal of Scientific Instrument $\mathbf{3 4}$ (2013),588-595.

[6] Mingxi Deng, Experimental study of evaluation of damage in metallic plates using electromagnetic acoustic resonance techique, Technical Acoustics 30 (2011),327-330.

[7] Nian Jiang, Zhaoba Wang, Youxing Chen, Yu Chen, The experiment parameters of the steel-sheet thickness measurement by electromagnetic ultrasonic, Chinese Journal of Sensors and Actuators 28 (2015),498-502.

[8] Zhichao Cai,Lan Chen,Zhenyong Zhao,Guiyun Tian, Research on electromagnetic acoustic resonance for plastic deformation in Q235steel, Chinese Journal of Scientific Instrument 40 (2019),153-160. 